\title{
Students' context-sensitive use of conceptual resources: A pattern across different styles of question about mechanical waves
}

\author{
Lisa M. Goodhew $\odot,{ }^{1}$ Amy D. Robertson, ${ }^{1}$ Paula R. L. Heron $\odot,{ }^{2}$ and Rachel E. Scherr ${ }^{3}$ \\ ${ }^{1}$ Department of Physics, Seattle Pacific University, Seattle, Washington 98119, USA \\ ${ }^{2}$ Department of Physics, University of Washington, Seattle, Washington 98195, USA \\ ${ }^{3}$ School of STEM, University of Washington, Bothell, Washington 98011, USA
}

(Received 14 February 2020; revised 19 February 2021; accepted 6 April 2021; published 21 May 2021)

\begin{abstract}
Resources theory assumes that resource activation is context sensitive, and that an important dimension of context is the question students are answering. The context sensitivity of resource activation has been demonstrated empirically by case studies that show students using different resources to answer questions that are similar in focus. In this paper, we further substantiate and add specificity to the field's understanding of the context sensitivity of resource use by demonstrating a pattern in resource activation for questions about mechanical pulse propagation, superposition, and reflection. In particular, our analysis shows a pattern in the kinds of resources students use to answer different styles of questions about the same physics topic. Questions that ask for a prediction tend to elicit rules or procedures, while questions that ask students to explain an observation elicit these plus ideas about force, energy, and motion. Our results call both researchers' and instructors' attention to the style of question that they ask and the impact it has on the resources commonly cued for students.
\end{abstract}

DOI: 10.1103/PhysRevPhysEducRes.17.010137

\section{INTRODUCTION}

The resources theoretical framework models cognition in terms of pieces of knowledge-i.e., resources - that are activated in the moment, in a context sensitive way, to construct arguments, models, and concepts [1-5]. However, few studies have explicitly investigated whether there are patterns in context sensitivity that bear out across many students, or whether certain features of the context reliably elicit particular kinds of resources. Resources research has validated theoretical claims of context-sensitivity primarily through vignettes and case studies that showcase a single student using different lines of reasoning in different situations [2,6-8]. For example, Wagner [8] illustrates context sensitivity with the story of Kristin, an undergraduate student who uses two different lines of reasoning to answer two conceptually isomorphic probability questions, one about flipping a coin and another about dropping a ball through a vertical pinball machine. Our study demonstrates a pattern of context sensitivity across many students' responses to conceptual wave mechanics questions.

Published by the American Physical Society under the terms of the Creative Commons Attribution 4.0 International license. Further distribution of this work must maintain attribution to the author(s) and the published article's title, journal citation, and DOI.
In this paper, we show that different styles of questions about the same wave mechanics phenomenon elicit responses that tend to draw on different types of conceptual resources at different frequencies-in particular, resources about forces, motion, and energy vs resources for problemsolving rules and procedures. Our data include students' written responses to several conceptual physics questions asked as part of a larger study (e.g., Ref. [9]) about students' conceptual resources for understanding mechanical waves. In that study, we asked conceptual questions that can be broadly characterized as two types: (a) questions that ask students to make a prediction about the outcome of an experiment or changes to an experiment, and then explain their reasoning for the prediction (predict-style questions), and (b) questions that describe the outcome of an experiment and ask students to explain why the outcome makes sense (explain-style questions). Here, we examine data from pairs of questions about three wave mechanics ideas: pulse propagation, superposition of pulses, and reflection of a pulse at a boundary.

In our initial examination of these data for another study [9] we noticed a pattern of strikingly different kinds of answers to predict-style and explain-style questions, which led us to more closely examine-and then seek to characterize-these differences. For example, one question about pulse propagation asked students to describe a change to a string that would result in a faster-moving pulse and to explain their answer (a predict-style question), while another question asked students to explain why it 
makes sense that increasing the tension in a spring results in a faster-moving pulse (an explain-style question). The responses shown in Figs. 1 and 2 illustrate the different kinds of responses to each type of question that stood out to us. The example in Fig. 1, which is a response to the predict-style question, states that increasing the tension in the spring will increase the speed of the pulse, and justifies this prediction using an equation for the speed of a pulse on a string. In contrast, the example in Fig. 2-a response to the explain-style question-describes the forces acting on a small part of a string as a pulse reaches it and constructs a mechanistic description of how increased tension affects the speed of the pulse. While both responses assert that pulse speed depends on tension, the reasoning used to justify this dependence is quite different. The first response invokes an equation and says how it applies to the particular situation, while the second causally explains how tension causes an increase in the speed of the pulse.

Our initial examination of the data left us with the sense that contrasts like these were common, and this led us to form the preliminary hypothesis that predict-style questions tend to elicit ideas that are rule-based (i.e., involving statements of fact, principle, or problem-solving procedure), while explain-style questions elicit more

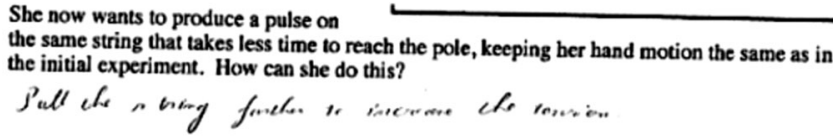

Explain your reasoning for your answer.

$v: \sqrt{\frac{f_{I}}{\mu}} \leadsto \uparrow F_{T}=\uparrow v$

FIG. 1. Example student response to a predict-style question. "[to make a pulse that takes less time to reach the pole, she should] pull the string further to increase the tension [because] $v=\sqrt{ }(T / \mu)$ and $\uparrow F_{T} \rightarrow \uparrow v$." mechanistic ideas (i.e., involving chaining ideas in a semi-causal manner [10]). In this paper, we describe the process by which we sought to test this hypothesis: we developed a coding scheme that articulates the difference between these two types of responses, and applied it to a large number of responses to predict-style and explain-style questions in order to quantify our initial observations. Our results both affirm and add nuance to our initial hypothesis.

\section{RESOURCES THEORETICAL FRAMEWORK}

Resources theory highlights the complex, dynamic, and emergent nature of student thinking [11] and emphasizes the continuity of that thinking with formal physics concepts $[1,4,5,12-14]$. Our work draws on the resources theoretical framework to identify patterns in student thinking about mechanical waves, particularly by emphasizing the context sensitivity of student thinking.

The written responses we analyze in this study are evidence of a collection of resources activated for each student as they answered our questions. Individually, these resources may vary in their grain size and level of sophistication, and they may be activated in clusters to construct arguments or concepts $[6,15]$. However, the focus of this study is on the kinds of resources evidenced by students' written responses, rather than on individual resources themselves. Consistent with resources theorywhere learning physics involves activating and building from the resources that already exist in the student's mind, and can involve resources that are canonically incorrect or incomplete $[1,2,4,5,16]$ —we assume that both kinds of resources characterized by our coding scheme are valuable for physics learning $[2,4,6,12,13,16-18]$.

This study is most prominently informed by resource theory's prediction of context sensitivity in student thinking. Resources are activated when they "make sense" or are deemed applicable by the student; activation of a particular

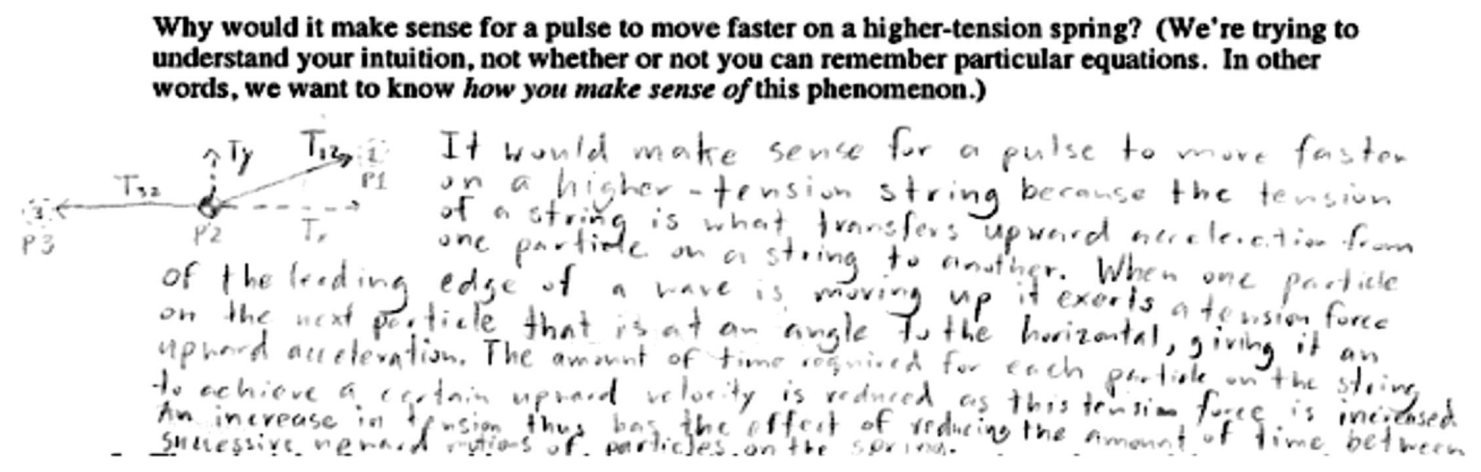

FIG. 2. Example student response to an explain-style question. "It would make sense for a pulse to move faster on a higher-tension string because the tension of a string is what transfers upward acceleration from one particle on a string to another. When one particle of the leading edge of a wave is moving up it exerts a tension force on the next particle that is at an angle to the horizontal, giving it an upward acceleration. The amount of time required for each particle on the string to achieve a certain upward velocity is reduced as this tension force is increased. An increase in the tension thus has the effect of reducing the amount of time between successive upward motions of particles on the spring." 
resource is influenced by the lived experience of the learner, the social setting in which the question is asked, and the question at hand $[2,6,17]$. Thus, resources theory does not view student thinking as stable, coherent, and consistently applied; instead it assumes that specifics of the context in which a question is asked will influence the resources activated in a student's mind [1,2,6,16,19]. At the same time, resources theory leaves open the possibility of predictable patterns of resource use, because patterns in the context may lead to patterns in the resources activated. In this study, we specifically attend to the question type as a dimension of context.

\section{QUESTION ADMINISTRATION}

In this study, we analyzed students' responses to conceptual questions about three mechanical wave phenomena: propagation, superposition, and reflection. For each of these topics, we analyzed one predict-style question and one explain-style question (as shown in Figs 3-8). These questions were asked as part of a broader study investigating students' conceptual resources for wave mechanics, where we began with predict-style questions that had been used in previous research and instruction and then modified these questions as explain-style questions to probe students' ideas more deeply. ${ }^{1}$

The propagation predict-style question was modified from Wittmann's Waves Diagnostic Test [21]. This question asked students to determine a change, either to the same string or by using a different string, that would make the pulse reach the end of the string faster (the original pulse-flick question, Fig. 3). The propagation explain-style question asked students why it would make sense that a pulse moves faster on a higher-tension spring (the tension pulse-flick question, Fig. 4). For both of these questions, a correct answer would indicate that pulse speed is determined by the tension and mass density of the string or spring. That is, pulse speed increases with increased tension and decreases with increased mass density. Answers to these questions can be justified in a variety of ways that are consistent with canonical physics thinking. For example: a statement that the speed of pulses and waves is determined by the properties of the medium they move through, an

\footnotetext{
${ }^{1}$ The text directing students to explain their thinking variede.g., "explain your reasoning" or "say why your answer makes sense to you" or "why is this the case?". In particular, the tension pulse-flick question included the text: "we're trying to understand your intuition, not whether you can remember particular equations. In other words, we want to know how you make sense of this phenomenon." We designed the tension pulse-flick question for another study, and we included this text because we wanted to know more about how students thought about the relationship between tension and pulse speed. Because the patterns we report here are repeated for questions that did not include this particular language, we think that they are cued by more than just this statement.
}

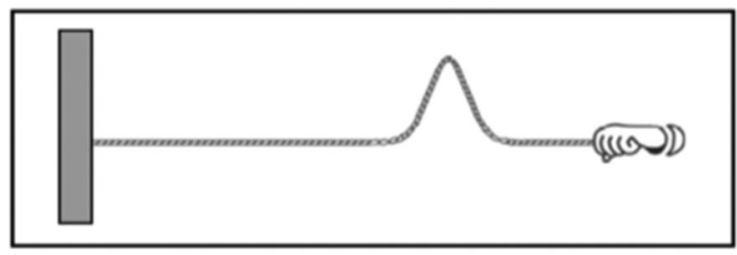

One end of a long taut string is tied to a distant pole and the other end of the string is held by a student (see figure above). The student quickly flicks her hand up and down to create a pulse moving toward the pole.

a. She now wants to produce a pulse on the same string that takes less time to reach the pole, keeping her hand motion the same as in the initial experiment. How can she do this? Explain your reasoning for your answer.

b. She still wants the pulse to reach the pole in less time by making a change to the string. How can she do this? Explain your reasoning for your answer.

FIG. 3. The predict-style question on pulse propagation (the original pulse-flick question). This question is modified from Wittmann [20].

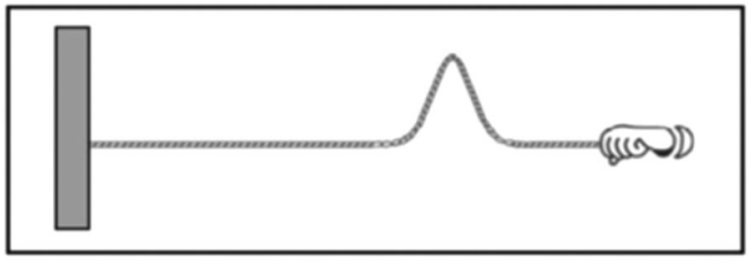

Consider the following two scenarios: In scenario 1, your Teaching Assistant (TA) creates a pulse by flicking the end of a spring, as in the figure above. In scenario 2, your TA pulls the spring so that it is more taut (i.e., increases the tension in the spring) and then creates a pulse by flicking the end of the spring in the same way. The pulse in scenario 2 travels down the spring faster (i.e., has a larger speed) than the pulse in scenario 1 . Why would it make sense for a pulse to move faster on a higher-tension spring?

FIG. 4. The explain-style question on pulse propagation (the tension pulse-flick question).

equation that quantifies the relationship between speed and properties of the medium, or a mechanistic description of how changing tension or mass density affects the motion of small parts of the medium and thus affects the speed of propagation. Section V includes student responses that illustrate these various types of explanations.

The superposition predict-style question (the pulse subtraction question, Fig. 5) presented students with a figure showing both (i) the shape of a spring at an instant when two pulses overlap and (ii) the shape of one of the pulses at that instant. This question asked students to draw the shape 

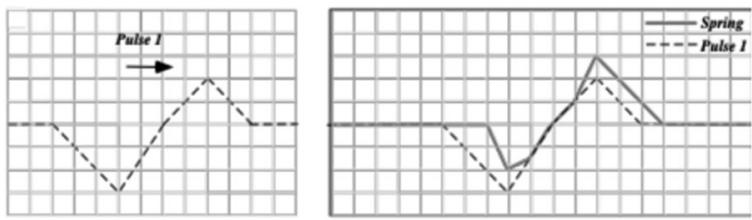

Pulse 1 moves to the right along a spring, as shown in figure (a) above. Another pulse (pulse 2, not shown) moves in the opposite direction along the same spring. Figure (b) above shows the location of pulse 1 (dashed line) and the shape of the spring (solid line) at an instant while the two pulses meet.

On Figure (b), draw the shape and location of pulse 2 at the instant shown. Say why your answer makes sense to you in the space below.

FIG. 5. The predict-style question on superposition (the pulse subtraction question). This question is modified from Kryjevskaia, Stetzer, and Heron [22].

of the second pulse at that instant, and to explain why their answer makes sense. We modified this question from Kryjevskaia, Stetzer, and Heron's previous study of student ideas on mechanical pulse superposition and reflection [20]. The superposition explain-style question asked students to explain why pulses add when they meet (the why superposition question, Fig. 6). For the pulse subtraction question, the correct shape of pulse 2 can be obtained by subtracting the displacement of pulse 1 from the displacement of the spring at each location along the spring. Analogously, the shape of the spring shown in the right diagram for the why superposition question is the sum of the displacements of the two pulses at each location along the spring. For both of these questions, there are a variety of ways to explain the shape of the spring that are consistent with canonical physics. These include a description of the procedure used to apply the principle of superposition to two pulses on a spring, a statement that pulses or waves do not behave like objects but instead are superposed when they coincide, or a mechanistic description of the additive effects of each disturbance on the part of the spring where
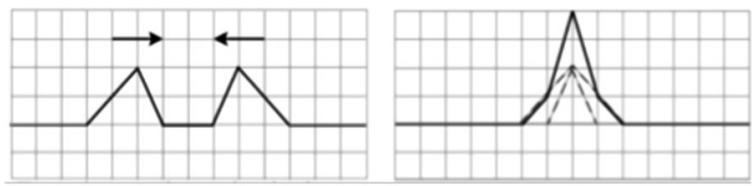

When two wave pulses on the same side of a spring meet, they add, as in the figure at right. This is an example of superposition.

How do you make sense of this phenomenon? In other words, why do pulses add when they're on the same side of the spring/why does the superposition principle work the way it does?

FIG. 6. The explain-style question on superposition of two pulses (the why superposition question).
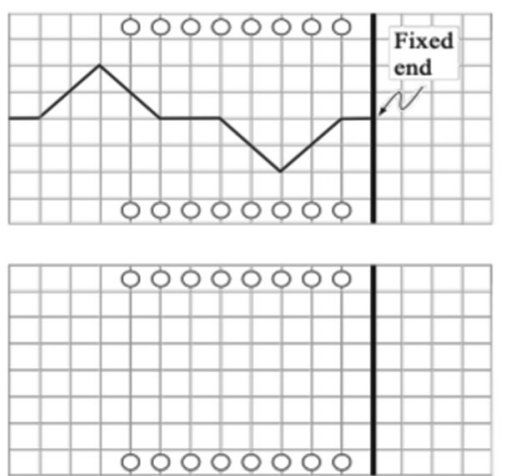

A pulse moves to the right along a spring with a fixed end. Cups are placed on either side of the spring as shown. The maximum transverse displacement of the spring while the pulse approaches the end of the spring is less than the distance from the equilibrium position of the spring to the cups. At the time shown, no part of the pulse has reached the fixed end of the spring.

On the diagram at right, mark which (if any) cups are knocked over during the reflection of the pulse from the fixed end. Briefly explain.

FIG. 7. The predict-style question for reflection (the cups question). This question was modified from Kryjevskaia, Stetzer, and Heron [22].

they meet. Student responses that illustrate these lines of reasoning are given in Sec. V.

The reflection predict-style question asked students to predict which of several paper cups, arranged on either side of a spring near its fixed end, will be knocked over when the depicted pulse reflects at the endpoint (the cups question, Fig. 7). We modified this question from Kryjevskaia, Stetzer, and Heron's previous study of student ideas on mechanical pulse superposition and reflection [18]. The reflection explain-style question asked students to explain why a pulse reflects on the opposite side of a spring at its fixed end (the why reflection question, Fig. 8). For the cups question, the shape of the spring can be determined by modeling the boundary as a point that is equidistant from the leading edge of the incident pulse and the leading edge of another pulse, traveling in the opposite direction toward the boundary, that is the same shape as the incident pulse but reflected across the $x$ and $y$ axes (which we refer to as the "ghost pulse"). The displacements of the two pulses add according to the principle of superposition during the time interval that they overlap. When displacement of the spring (on the left side of the boundary) meets or exceeds the location of the cups, it is knocked over. For the situation in Fig. 7, only the second cup from right, above the spring, is knocked over. The same procedure can be used to determine the shape of a spring at several instants during the reflection of a single pulse as shown in the figure of the why reflection question (Fig. 8). For both questions there are a variety of ways to explain the shape of the spring during 

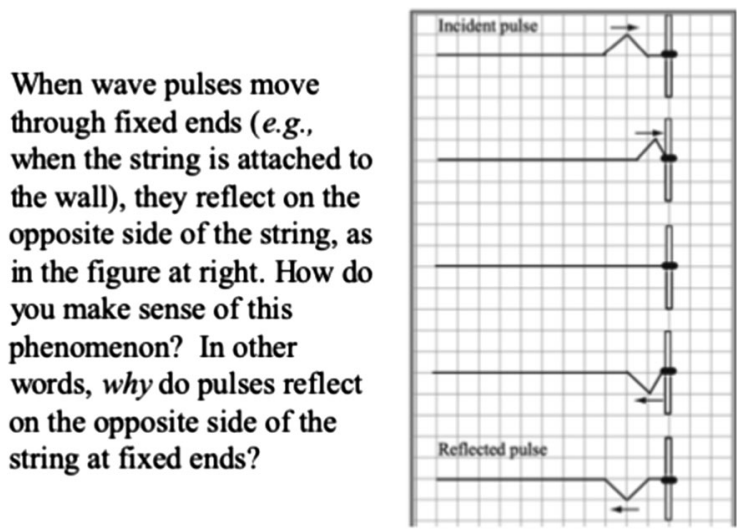

FIG. 8. The explain-style question for reflection (the why reflection question).

reflection that are consistent with canonical physics, such as describing the model used for reflection at a fixed end, explaining the procedure used to determine the shape of the pulse as it reflects, or by articulating a mechanism that causes the pulse to reflect at the fixed end. Examples of responses using each of these lines of reasoning are illustrated in Sec. V.

In total, our data for this investigation include 2102 student responses spanning a total of 17 courses from 5 universities in the US. For each question described above, we analyzed student responses from 2-3 universities. All of these courses were introductory, calculus-based physics courses that covered wave mechanics, ${ }^{2}$ though the specific topics and depth of coverage varied from course to course. In all cases these questions were given after instruction on wave mechanics and were included on homework (extra credit in one case), quizzes, or exams. In some courses, these questions were graded for correctness, in some they were graded for correctness and correct explanation, and in some they were graded for participation. We expect that specific grading practices and expectations varied across the courses in which we asked these questions, but we do not know exactly what they were. Table I gives additional details about the administration of these questions.

The demographic composition of our study sample may limit the extent to which our results apply to a representative introductory physics course. As Kanim and Cid point out [22], physics education research (PER) has historically oversampled from white, wealthy, mathematically prepared populations of students. They write,

"While Latino/Latina, Black, and Indigenous American students are $34.5 \%$ of the college-bound students taking the SAT, only $15.2 \%$ of the [physics education] research student population are from these groups."

\footnotetext{
${ }^{2}$ A typical introductory university-level physics course in the US includes instruction on wave propagation, superposition, and reflection, as well as wave optics and interference.
}

TABLE I. Details of the samples used in this study. At University B, our questions were given in multiple independent sections of the course taught by different instructors. Multiple questions were given in a course at University A and in some courses at University B.

\begin{tabular}{lccc}
\hline \hline $\begin{array}{l}\text { Question } \\
\text { administered }\end{array}$ & University & $N$ & $\begin{array}{c}\text { Fraction of course } \\
\text { participating }\end{array}$ \\
\hline Propagation & $\mathrm{B}$ & 70 & 0.97 \\
$\quad$ predict style & $\mathrm{D}$ & 134 & 0.76 \\
Propagation & $\mathrm{B}$ & 163 & 0.91 \\
explain style & $\mathrm{E}$ & 105 & $\sim 0.7$ \\
& $\mathrm{D}$ & 162 & 0.7 \\
Superposition & $\mathrm{A}$ & 175 & 0.91 \\
predict style & $\mathrm{B}$ & 153 & 0.85 \\
& $\mathrm{C}$ & 65 & 0.94 \\
Superposition & $\mathrm{A}$ & 170 & 0.89 \\
explain style & $\mathrm{B}$ & 76 & 0.9 \\
& $\mathrm{D}$ & 200 & 0.87 \\
Reflection & $\mathrm{A}$ & 99 & 0.79 \\
predict style & $\mathrm{B}$ & 24 & 0.64 \\
& $\mathrm{D}$ & 140 & 0.78 \\
Reflection & $\mathrm{A}$ & 169 & 0.8 \\
explain style & $\mathrm{B}$ & 69 & 0.96 \\
& $\mathrm{D}$ & 128 & 0.73 \\
\hline \hline
\end{tabular}

This is compared to white students comprising $62.9 \%$ of the PER student population and only $45.3 \%$ of collegebound students taking the SAT. In spite of this disproportionality, the PER community has historically treated the results of PER as indicative of "typical" university students, as though our samples are representative of the population of introductory physics students at large. This unexamined assumption means not only that the benefits of decades of research are disproportionately skewed toward mathematically prepared, white, wealthy populations of students but also that there is a higher likelihood of racialized bias in research-informed physics teaching and learning, as students are being implicitly assessed against a "norm" that was constructed from largely white, wealthy students [23,24].

Following the methods in Kanim and Cid, we approximated the demographics of the introductory physics courses in our study using university-level data, and then constructed a weighted sample based on the numbers of students in each course. Ideally, we would compare (a) the demographics of our study to (b) the demographics of a representative introductory physics course. However, we know neither (a) nor (b)-i.e., we did not collect samplelevel demographic data, and, as of the writing of this manuscript, we (as a PER or physics community) have not done the work to identify what counts as a representative sample of introductory physics students [25]. What we have done here allows for a coarse comparison similar to the one done by Kanim and Cid. 
$60.00 \%$

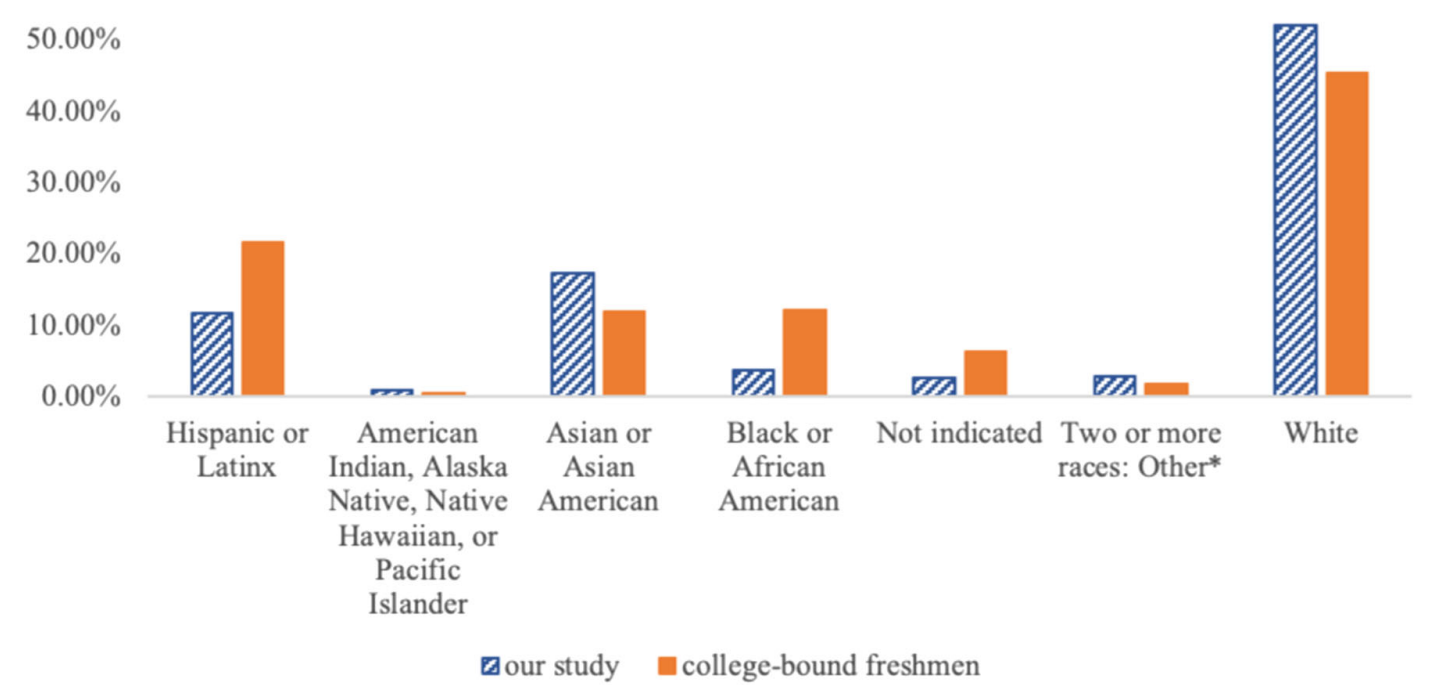

FIG. 9. Racial and/or ethnic demographics of our sample versus college-bound freshmen. Orange bars were constructed from percentages cited in Kanim and Cid [22]; blue striped bars were constructed from publicly accessible university-level demographic data, weighted by sample size.

As Figs. 9 and 10 show, this study likely oversamples from white, Asian, and wealthy student groups and undersamples from Latinx, Indigenous, Black, and low-income student groups. We say "likely" because results from earlystage research being conducted by our team suggests that the demographics of introductory physics courses do not match institution-level demographics [26]. However, whether or not our research over- or undersamples from specific racial and/or ethnic groups, or from specific parental income quartiles, as compared to a representative introductory physics course, we can say this: Figures 9 and 10 show that our sample overrepresents the physics ideas of white, Asian, and wealthy students, as compared to the college-aged population, and under-represents the physics ideas of Latinx, Indigenous, Black, and lowincome student groups. Whether this is a matter of who is being recruited into physics courses or a matter of sampling does not change that our results are limited in their generalizability in this way, and thus contribute to the systemic problems that Kanim and Cid highlight. This is a

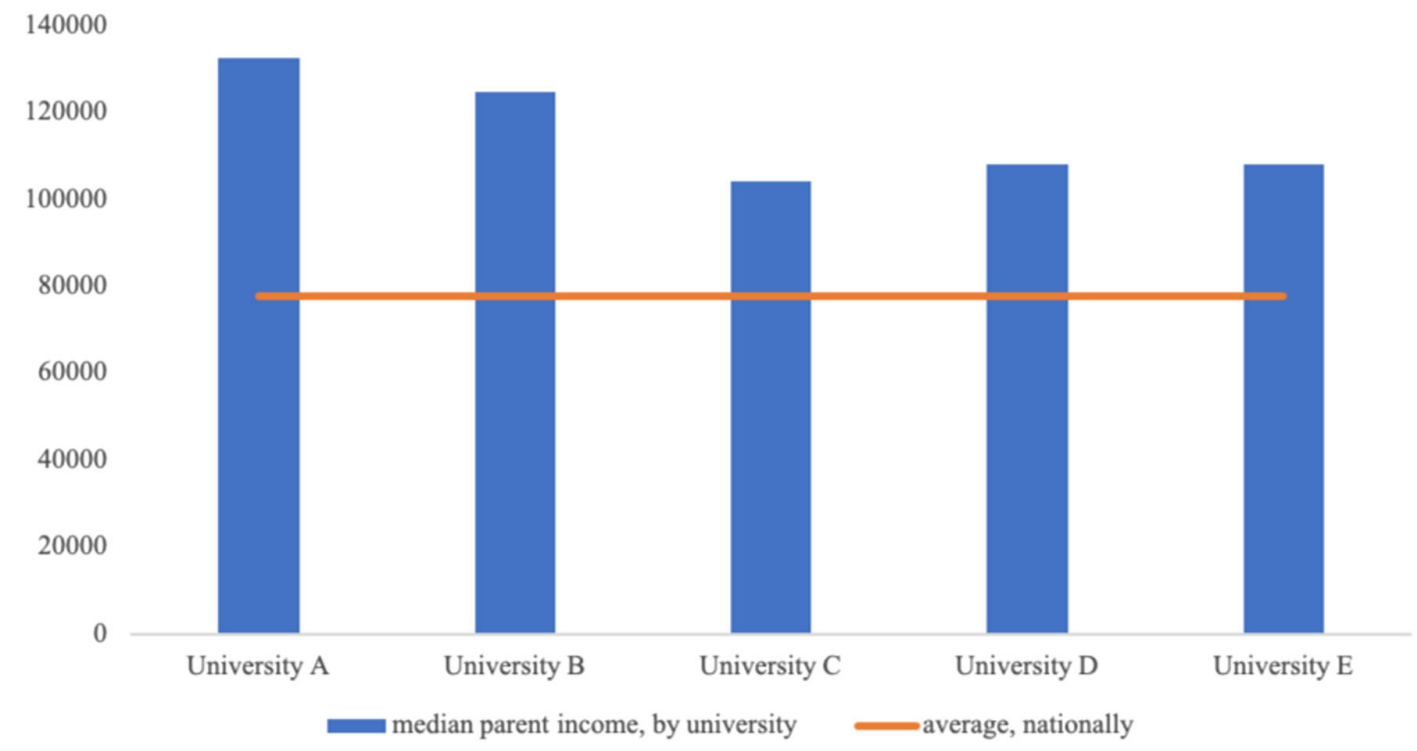

FIG. 10. Wealth demographics of our sample versus national average. Median parent income is in U.S. dollars. Blue bars were constructed from university-level data reported by the Equality of Opportunity Project [33]; the orange line represents the average of all median parental incomes reported there. 
community-level concern, not a manuscript-level one, and we raise it here, while also pointing out to recent worksuch as that done by Rosa [27], Hyater-Adams [28], Hazari [29], Rodriguez and Zamarripa-Roman [30], Quichocho [31], the PoC in PER group [32], and others-that can guide our conversations.

\section{METHODS}

This investigation was motivated by our initial observation that explain-style questions seemed to elicit more mechanistic responses, whereas predict-style questions seemed to elicit more rule-based or procedural responses. We began the process of constructing a coding scheme to refine this observation by roughly characterizing the kinds of responses that exemplified each category of resource and then articulating in detail what we meant by the two categories. This process led us to refine our sense of these two categories. Most significantly, we noticed that responses we initially labeled "mechanistic" tended to use ideas about force, motion, and/or energy in a loosely causal way to explain wave phenomena. We shifted our coding scheme to better capture this.

Our final coding scheme included two codes: (i) resources related to forces, motion, and energy (FME), and (ii) resources related to rules and procedures for modeling wave propagation, superposition and reflection (RP). The first code (FME) encompassed resources that described force interactions between parts of the string, pulse, or boundary; that described details of the motion of the string and/or boundary; or that described energy conservation, transfers, or transformations in the string system. The second code (RP) encompassed resources related to models or rules for propagation, superposition, or reflection, and resources regarding procedures for solving propagation, superposition, and reflection problems. We applied these codes to the ideas (typically at the sentence level) we noticed in students' responses. For example, we coded the example in Fig. 1 as RP because it uses an equation for the speed of a pulse on a string to justify a prediction that increasing tension will increase the speed of a pulse. (Although tension-a force-is mentioned, we do not see evidence of resources about forces or how they affect the motion of objects in this response.) We coded the example in Fig. 2 as FME because it uses ideas about the forces acting on a part of the medium and how these forces affect the motion of that part of the medium. The two categories are illustrated in detail in the following section.

For the sake of establishing clear bounds for each of these codes, we chose to only code RP or FME ideas that we, as physicists, recognized as relevant (even if incomplete or incorrect) for wave mechanics questions. Our coding scheme captures resources that we understand to be valuable for answering the physics question at hand (i.e., that are disciplinarily productive [17]), but does not necessarily capture all of the resources that may be valuable for students toward an emergent conceptual goal (i.e., that are situatedly productive [17]). This is primarily a practical choice: in static written data, we cannot see how the ideas expressed contribute to a student's own unfolding sensemaking process or self-defined questions, but we can see how these ideas are relevant for the physics questions at hand. This means that there are likely rules or procedures (or force, motion, or energy ideas) that are not captured by this coding scheme because our perspective as physicists limits our ability to recognize how they are relevant for students' own emerging understandings of wave mechanics. We do not expect that our categories capture all of the resources that students bring to bear in answering these questions, and they are not meant to indicate that these are the only types of thinking that researchers or instructors should attend to. However, as we will show in the next sections, these two categories of resources are relatively common (at least in some questions), represent rich physics thinking, and answer to our original purpose of testing our hypothesis.

We chose not to assign codes to responses that were in a "gray area" between the two codes. For example, we did not code the response, "pulses are reflected because "every action has an equal and opposite reaction," because as a rule this idea not (to us) directly relevant for pulse reflection, and it is not clear to us whether the action in this case is the pulse itself, or a force exerted on the spring that makes it move in a particular way, or something else entirely.

Two researchers (authors L.M.G. and A.D.R.) independently coded student responses to each question using the refined scheme. A single response could receive no codes, one code, or both codes (the latter if students issued separate lines of reasoning). We used Cohen's kappa as a measure of the interrater reliability of our codes [34]. This statistic improves upon simple measures of percentage agreement by taking into account chance agreement between the two coders. Our kappa values were 0.84 for the RP codes and 0.86 for FME codes. Kappa values in this range are typically understood as "strong" or "excellent" agreement $[35,36]$. Because our agreement was very high, we did not further refine our coding scheme after this step.

In reporting the percentages of student responses that used each type of resource (Sec. V), we kept only codes that were assigned by both coders. The final codes assigned to a single response reflect complete agreement between coders.

\section{TWO CATEGORIES OF CONCEPTUAL RESOURCES}

In the following sections, we give examples that show the variety of resources and responses encompassed by each of our categories (RP and FME), and we describe the bounds of these codes. 


\section{A. Rules and procedures category}

Across questions, students invoked rules and procedures to explain or justify their answers. The rules and procedures included in our scheme are specific to mechanical wave superposition, propagation, and reflection (e.g., pulses reflect on the opposite side at a fixed end), including principles and predictive models for the behavior of waves, equations for wave mechanics, and procedures for determining the outcome of experiments.

\section{Rules}

"Rules" responses often take the form of "if-then" or "when $x$ then $y$ " statements. These responses may connect specific salient features of the given situation to specific outcomes or behaviors, or they may state a principle or models as given. Some responses apply a physical model to the specific question being asked, as in these responses:

"A fixed end can be modeled by a reflected, inverted pulse approaching from the opposite side of the boundary. I superimposed the incident wave shifted $5 \mathrm{~m}$ right and the imaginary wave shifted $5 \mathrm{~m}$ left." (cups question)

"Inversion occurs when the transmitted pulse moves towards a medium with a greater mass density. The fixed end always has a greater mass density than the medium through which the transmitted pulse travels so inversion will occur." (reflection mechanism question)

"The speed the pulse is moving at is a property of the spring. This means that if you were to change something about the spring, then the velocity will change. If we assume the frequency is going to remain the same, then the pulse is going to need to travel at a faster speed so it can cover a lager distance in the same amount of time as the shorter distance." (tension pulse-flick question)

The first response highlights that a salient feature of the situation is the fixed end of the spring, and describes the model that applies in fixed-end situations (that the reflection is like a superposition of the incident pulse, and a matching "ghost pulse" is coming from the opposite side of the boundary and on the other side of the spring). The response then describes how the model is applied to the particular situation using superposition, which instantiates another facet of the RP category which we discuss below. The second response above states a different rule-that pulses invert when they reflect at a boundary with a denser medium - and applies the rule to the why reflection question. The third response above states as a rule that pulse speed is a property of the medium it travels through and describes how this rule applies to the situation in the tension pulse-flick question.

We included in our coding rules that were relevant for the question, even if they were not canonical principles or models for the situation, or if the model was applied incorrectly. For example:

"I think this happens because...as the wave moves into a fix[ed] end it is like any other object hitting a wall that would be reflected in the same angle but opposite side." (why reflection question)

The rule that objects reflect at an equal and opposite angle to the incident is relevant for reflection, which was the topic of the question. Thus, we assigned this response the RP code even though the mechanics of a Newtonian object reflecting at a barrier are different than the mechanics of a string as a pulse reflects at its fixed end.

\section{Equations}

Particularly in the propagation questions, some responses stated an equation as a justification or explanation, such as these examples:

"Ito make a pulse that takes less time to reach the pole, she should] pull the string further to increase the tension [because] $v=\sqrt{T / \mu}$ and $\uparrow F_{T} \rightarrow \uparrow v$." [see Fig. 1] (original pulse-flick question)

"[The pulse] moves faster [on a tauter spring] because $v=\sqrt{T / \mu}$; if the linear density of the string is lower, then $v$ will be higher. Pulling the string tighter lowers the linear density." (tension pulse-flick question)

"If she moves her hand in the same motion as she did originally but just flicks it faster it will take less time to reach the pole, [because] $v=\lambda f$ so when she flicks her hand quicker frequency is higher than before so velocity is faster." (original pulse-flick question)

Each of these responses states an equation that applies to pulses or waves on a string or spring and explains how the equation applies to the particular situation (e.g., pulling the string tighter increases $T$ or decreases $\mu$ ) and what the equation predicts (e.g., pulse speed increases). Responses like these use a model in a particular way-by explicitly stating an equation - to justify a prediction or answer. Like responses that use other forms of models or rules, these responses tend to state the rule as given or as a premise of the student's argument.

\section{Procedures}

Some responses in this category describe the steps used to make a prediction or the mathematical procedure used to determine an answer.

"By the principle of superposition, wave_result $=$ wave_ 1 + wave $\_$, or wave_2 = wave_result - wave $\_1$. This subtraction is done at each point along the line." (pulse subtraction question) 
"I went point by point to make the second pulse add or cancel out with pulse 1 so the spring is the result of the two." (pulse subtraction question)

"I got my results by drawing my diagram as if it could keep continuing through space but anything after the fixed end was reflected on the other side. I then added the amplitudes to any areas that intersect and got my result." (cups question)

The first two responses describe a procedure for applying the principle of superposition to the pulse subtraction question - the amplitude of the given pulse is subtracted from the shape of the spring shown at each point along the line. Similarly, the third response describes the mathematical procedure (adding amplitudes of the parts of the pulse that overlap as it reflects) that is used in conjunction with the "ghost pulse" model to answer the cups reflection question. Common to all three responses is that they describe the steps that the student used to determine the answer to the question.

Other responses referenced a mathematical procedure for determining the answer without describing the steps, such as these examples:

"By superposition, the amplitudes will add." (cups question)

"This was very hard to visualize, but, it seems that the spring position would just be the average of the two waves' positions." (pulse subtraction question)

The first response references the principle of superposition to justify that the amplitudes of two pulses (which we assume are the incident and reflected parts of a pulse reaching the fixed end of the string in the cups question) add together. It also references a procedure (addition) for determining the answer. The ideas expressed in this response could be called either a "rule" or a "procedure;" our aim is to show the variety within the RP category, rather than to emphasize distinctions between various kinds of responses within the category. The second response cites a mathematical procedure - averaging - to justify an answer. Though averaging is not the correct mathematical procedure for the pulse subtraction question, it is a sensible mathematical procedure for this situation, and thus we include it in the RP category.

In summary, we grouped these rules, equations, and procedures into one category because they represent succinct, specific ideas, that can be used to make predictions about wave propagation experiments.

\section{B. Force, motion, and energy category}

Particularly for the explain-style questions, many responses mapped fundamental mechanics ideas onto wave phenomena. Responses in this category described forces on the pulse or medium or between parts of the pulse or medium, described motion of parts of the pulse or medium, or used energy conservation or tracking to make sense of wave phenomena.

\section{Forces}

In some responses, students explained or predicted a phenomenon by describing the forces acting on or between parts of the system, as illustrated in the following examples:

"Every action has an equal and opposite reaction. So as the wave propagates towards the wall, and hits the wall, there is a force upwards on the wall. This means the wall exerts a force downwards on the string. Because $F=$ $m\left(\frac{d v}{d t}\right)$ and the wall does not accelerate, the string accelerates downwards, effectively inverting the wave. Also, maybe something to do with energy, I D[on't] K[now]." (why reflection question)

"The principle of superposition can be explained by looking at the forces acting on individual particles of the spring. Looking at a single particle, the forces acting on it will be from the adjacent particles right next to it. The net forces will be from the adjacent particles right next to it. The net forces will vary depending on where the particle is on the spring. Once two wave pulses meet that are on the same side, the net force will be the sum of both waves causing a higher magnitude of force, therefore a higher displacement." (why superposition question)

"By increasing the tension, we increase the contact forces between particles of the string, thus they do not have to displace far to transmit the motion. It takes less time for each particle to transmit [the] pulse." (tension pulse-flick question)

Each of these responses describes how forces acting on or between parts of the medium cause the observed behavior of the string or pulse. The first response applies Newton's second and third laws to the part of the string near the boundary (the wall): the pulse is inverted because the string pulls up on the boundary, and the boundary pulls down with an equal and opposite force, which causes the string to accelerate and move past the equilibrium point. The second and third responses describe how force interactions between small parts of the medium cause the undisturbed parts of the medium to be displaced, and the magnitude of the force determines how high (in the second response) or how quickly (in the third response) the medium is displaced from equilibrium.

As illustrated by the examples above, many of the responses we assigned this code were quite detailed, but the level of detail was not a criterion we used to determine which responses to assign the FME code. Other examples, like this one, more briefly referenced forces in the medium in their answers: 
"It makes sense because the forces in the pulses that cause the displacement, just like with basic vector math.” (pulse subtraction question)

This response implies that when two pulses are co-located, the forces associated with each pulse add as vectors to cause the observed displacement of the medium. This response is not specific as to whether the forces act on the pulse as a whole or on parts of the pulse. However, like other force examples in the FME category, the basis of its argument is that forces "in" the pulse cause the observed phenomenon (superposition of two pulses, in this case).

\section{Motion}

Other responses in the FME category examined motion of parts of the medium or parts of the pulse to construct an explanation, as the following examples show:

"For each wave pulse, the pulses have vertical velocities which have the same direction, when they meet each other, the vertical velocities combine and give a larger vertical velocity." (why superposition question)

"I just think of it as a wave on top of a wave. A wave displaces a medium. If that medium is already displaced, the wave will still act the same way." (why superposition question)

“... I think this happens because it is a fixed end and because the pulse is not allowed to drift upwards to meet its highest peak at the boundary point, it is pulled down and continues with this motion of downward motion when reflected and will then stay on that side because nothing is pushing it back up to the origin." (reflection mechanism question)

"[A] tauter string means a greater length is affected at once by the movement (longer $\lambda$ )." [accompanied by diagram in Fig. 11] (tension pulse-flick question)

The first and second examples here explain that two pulses add when they are colocated because each pulse makes the medium move away from its equilibrium position, and the effects on the motion of the medium from each pulse combine or add. The third response describes the transverse motion "of the pulse" during reflection in order to explain

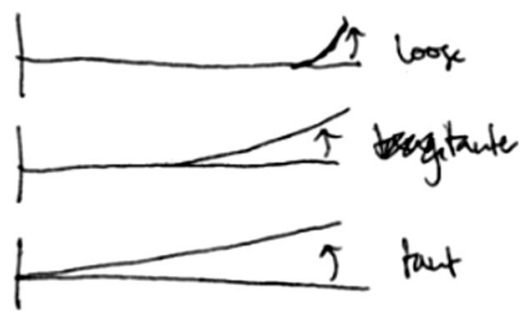

FIG. 11. Student drawing accompanying the response "[A] tauter string means a greater length is affected at once by the movement (longer $\lambda$ )." why a pulse is reflected on the opposite side at a fixed end. The fourth response above explains that when tension in a string is higher, more of the string is disturbed "at once" (i.e., as the source itself moves), and therefore the pulse is wider and must move faster. Though it is quite different from other examples of this category, this response still describes the motion of the medium and relates it to the motion of the pulse.

Other responses seem to describe a relationship between the motion of the pulse and the motion of the medium, or the transverse and longitudinal motions associated with the pulse:

"Repeat the same motion, but more quickly [because] the time it takes for the pulse to pass a certain point, leading edge to trailing edge (relates to velocity), is the same as the creation time of the pulse. By repeating the same motion (flick up and down) faster, the pulse will be faster." (original pulse-flick question)

"With more tension in the spring, there would be less of an amplitude, meaning the wave will travel less in the "up and down" motion and more in the "left to right" motion. Less tension will result in waves that have a greater amplitude (speed??)." (tension pulse-flick question)

The first response explains that a pulse that passes over each point on the string in less time will have a higher speed. While this is incorrect, because it does not account for the width of the pulse (or how many other particles of the medium are moving during the time a pulse passes a particular point), it appropriately describes pulse propagation as sequential transverse movements of parts of the medium. The second response above explains that if transverse displacement associated with the pulse is limited by tension, then there can be more longitudinal motion. Again, though this explanation is incorrect-transverse motion and longitudinal motion associated with the pulse are not the same kind of thing-we recognize it as explaining propagation in terms of motion of parts of the pulse and medium.

\section{Energy}

Some responses in the FME category explain or justify an answer using ideas about energy conservation or tracking, like these examples:

“... I believe that the reason it reflects to the other side is due to conservation of energy. Because the wave constantly wants to displace upwards, however [it is] unable to b/c of [the] fixed end. The only way it can go to maintain its energy is to deflect downward. Furthermore, energy has no direction, nor does it care about direction, therefore reflecting downwards does not change the energy state." (why reflection question) 
"Pulses reflect on the opposite side at fixed ends, because energy is lost during contact with the wall, causing the reflected wave to have less potential energy." (why reflection question)

"Pulses add because each wave or pulse is energy being carried across the spring (or other medium). When the 2 pulses cross each other, the resultant is the sum of their amplitudes because both energies are present at the same point at the same time." (why superposition question)

"Each part of the spring is pulling harder (in better contact) with the part next to it, so the energy transfers better or faster and the wave travels faster." (tension pulse-flick question)

The first response explains that there must be a reflected pulse to conserve energy in the system. This response does not say why the fixed end means that the pulse must be inverted in order for energy to be conserved; this is stated as given. The second response explains that a pulse must invert when it reflects at a fixed end because energy is lost during the reflection. We infer that the student sees the inverted pulse as a lower energy state, perhaps because the reflected pulse in the figure in this question is on the "bottom" of the string, and thus can be thought of as lower or having less gravitational energy. Although this is not a canonical explanation for the inversion of a pulse at a fixed boundary, this response instantiates the force-motionenergy category because it uses energy conservation to explain the phenomenon. The third response above explains that pulses carry energy across the medium, and thus when two pulses meet, the energy associated with each pulse is in the same location. This response implies that greater displacement from equilibrium indicates more energy at that location, and thus when the two pulses are colocated the medium is displaced more. The fourth response focuses on the energy transfers between parts of the medium as the pulse propagates. It identifies tension as a mechanism of energy transfer (i.e., the tension between parts of the spring) and associates the transfer of energy across the medium with the motion of the pulse itself, and uses these ideas to explain the observation that increasing tension in a spring increases the speed of a pulse moving on it.

We coded energy tracking or conservation ideas, but not statements that describe the pulse as energy. When students say "the energy of the pulse moves faster" we did not code it because this is not an explanation based on energy tracking or conservation. We grouped responses that involve force, energy, and motion ideas together into one code because they share an explanatory nature: these describe the physical entities and actions that are relevant in various wave propagation phenomena. In this sense, many of these responses share characteristics of mechanistic reasoning [10,37]. However, "mechanistic reasoning" was not the primary criterion for this code; there were causal statements in our data set that we did not assign the FME code because they did not clearly demonstrate force, energy, or motion resources. For example, the response: "In the second scenario, the spring is higher in tension, so it's less dense. And since it's less dense, the pulse can move along the string easier, therefore faster," (tension pulse-flick question) causally connects the density of the spring to the speed of the pulse and mentions tension, but does not use resources for forces, energy, or motion to describe the motion of the medium or propagation of the pulse. This criterion represents a refinement of our initial sense that this category was based on mechanistic resources.

In summary, the FME category encompasses resources for broadly applicable physics concepts that have been applied to wave mechanics questions, whereas the RP category encompasses resources that relate to the rulelike application of particular models, principles, and equations for wave mechanics. The example responses presented in this section illustrate two categories of conceptual resources which are, at least in the context of wave mechanics, ${ }^{3}$ distinct.

\section{PATTERNS IN FREQUENCY}

Figures 12-17 represent our coding of student responses to each question; each figure shows the fraction of responses from each university that we assigned ruleprocedure or force-energy-motion codes for each question. Since a single response could be assigned no codes, one code, or both codes, the fractions for a single university and question do not necessarily add up to 1 . These fractions include only the responses assigned a particular code by both coders. Across the six bar graphs, we notice a consistent trend: that predict questions elicit primarily rule-procedure resources, while explain questions elicit both rule-procedure and force-motion-energy resources. These results add nuance to our initial hypothesis that explain-style questions commonly elicit one kind of resource, while predict-style questions commonly elicit another.

More specifically, for each of our predict-style questions (Figs. 12-14), at least 50\% of responses used ruleprocedure resources, and, with the exception of the sample from University B given the original pulse-flick question, fewer than $2 \%$ of responses used force-energy-motion resources to answer predict questions. Across all samples given the explain questions (Figs. 15-17), both rulesmotion resources and force-energy-motion resources are relatively common. Each resource was used by more than

\footnotetext{
${ }^{3}$ We suspect that for kinematics and dynamics questions (i.e., questions about force, energy, and motion) these two categories may not be distinct, or there may be corresponding categories appropriate for the topic.
} 




FIG. 12. Fraction of responses assigned the RP (striped blue bars) or FME (solid orange bars) codes for the predict-style question on propagation (the original pulse-flick question).

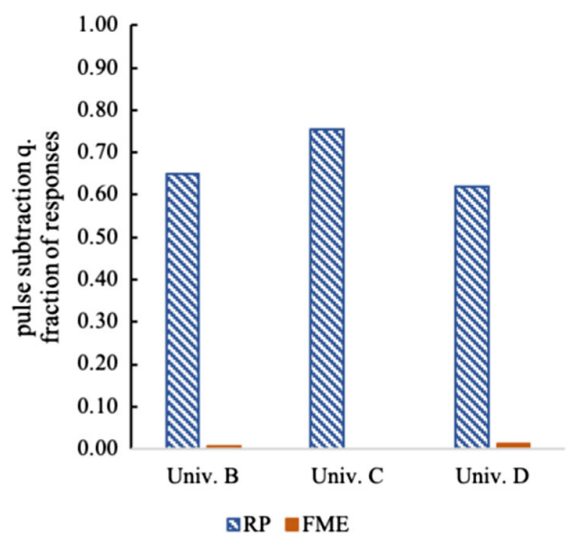

FIG. 13. Fraction of responses assigned the RP or FME codes for the predict-style question on superposition (the pulse subtraction question).

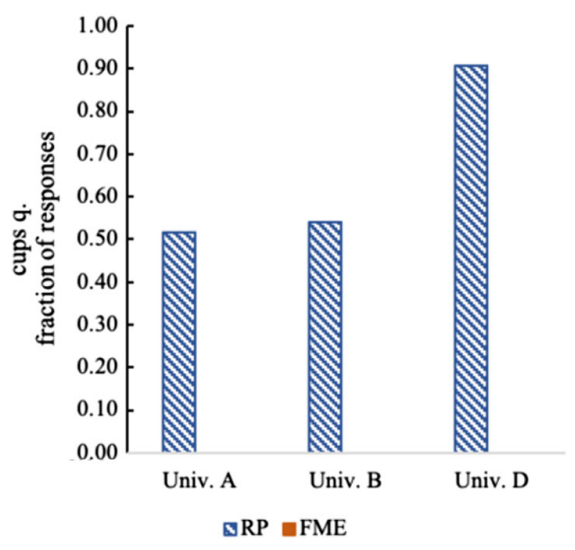

FIG. 14. Fraction of responses assigned the RP or FME codes for the predict-style question on reflection (the cups question).

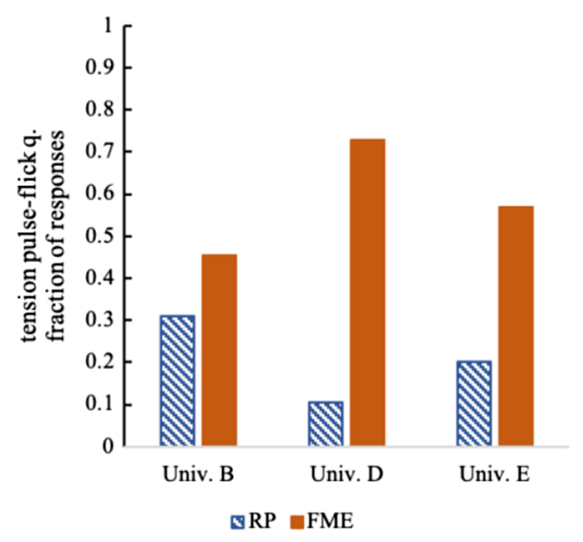

FIG. 15. Fraction of responses assigned the RP or FME codes for the explain-style question on propagation (the tension pulseflick question).

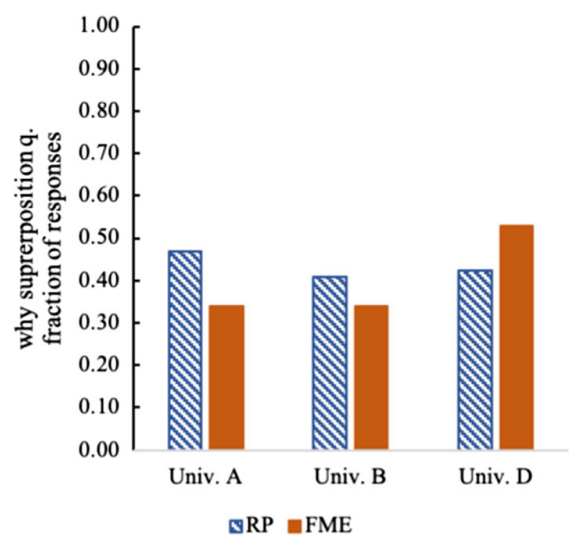

FIG. 16. Fraction of responses assigned the RP or FME codes for the explain-style question on superposition (the why superposition question).

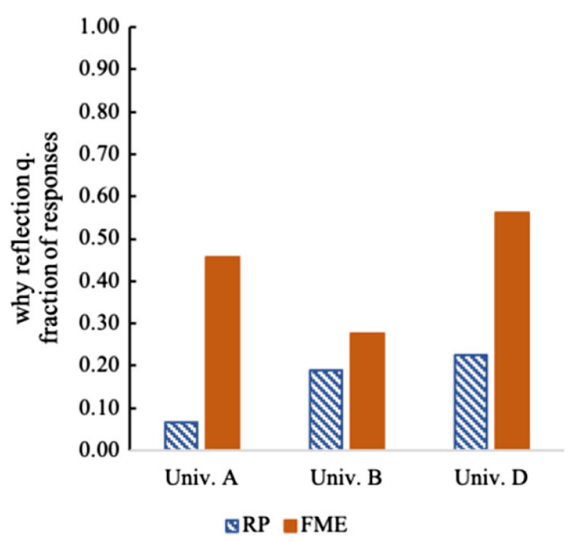

FIG. 17. Fraction of responses assigned the RP or FME codes for the explain-style question on reflection (the why reflection question). 
$20 \%$ of students in at least one sample given each question, and more than $5 \%$ of responses from every sample used each kind of resource. We note that overall, FME resources were more common than RP resources in these questions: FME resources were used in $28 \%-73 \%$ of responses to 'explain' questions, whereas RP resources were used in $7 \%-47 \%$ of responses. ${ }^{4}$

These patterns reproduce across universities and questions, with the exception of the original pulse-flick question at University B. The courses we sampled from were taught by different instructors, using a range of texts and course structures, and with varying content emphases. The fact that similar patterns emerge despite the variation in instruction suggests that the different patterns we see for the predict-style and explain-style questions can be made sense of, at least in part, by differences in the questions themselves. This latter claim draws on a model of generalizability that looks for patterns that reproduce across multiple sources of heterogeneity; that is, in the absence of a controlled experiment, the "operative question" becomes "can the same...relationship be observed" across contexts [38]? If the differences in resource activation and use were primarily due to differences in instruction or grading, rather than the question, we would expect that in courses where instruction was most similar, the two questions would elicit similar patterns in responses. For example, at University A, four questions were administered in three courses (the two superposition questions were given in the same course, and the reflection questions were given in two other courses) taught by the same experienced instructor, in the span of one year. All three courses used the same text and followed the same instructional sequence. However, we see that the patterns in responses at University A resemble the patterns at the other institutions, where there was more variation in instruction. Hence this counterargument does not fully explain the observed patterns.

\section{THEORETICAL SIGNIFICANCE AND DISCUSSION OF FUTURE WORK}

Other constructivist perspectives in physics education research, such as the alternative conceptions perspective (e.g., Refs. [39,40]), have historically framed student thinking as stable and consistently-applied. Resources theory, on the other hand, predicts that students will not consistently apply the same line of reasoning to problems that a physicist would consider similar or even equivalent. While the context sensitivity of resources has been demonstrated in smaller samples through case studies, to our knowledge patterns in context-sensitivity have not been overtly explored on a large scale. Our results contribute to

\footnotetext{
${ }^{4}$ Of the three explain questions, the why superposition question most commonly elicited RP resources. This may be because the mathematical procedure of addition is particularly salient for understanding and explaining superposition.
}

the empirical validation of context-sensitivity using a large$N$ study and add specificity to theoretical notions of what features of the context matter for resource activation. In particular, this study suggests that the style of a question affects what kind of resources students use to answer it. The categories we articulate in Sec. V show that this pattern is more than students giving explanatory answers to explainstyle questions, and using models to make predictions. We see that there are specific ideas-force, motion, energy-that students commonly draw on to explain physics phenomena. We also see that students are drawing on rules, principles, and procedures relatively frequently, even as they explain physical phenomena.

One explanation for the patterns of context sensitivity we observe is that predict-style and explain-style questions cue different epistemological resources or framings, ${ }^{5}$ which in turn cue different kinds of conceptual resources for mechanical waves. Resources theory proposes that activation of epistemological and conceptual resources are linked, such that epistemological framing is expected to influence the activation of conceptual resources $[2,6,7,16]$. For example, a question on a worksheet in a physics classroom may either cue epistemological frames of "making common sense" or of "applying formal knowledge" for different students [2]. The making common sense frame in turn may cue conceptual resources related to everyday experiences that are appropriate for sense-making, while the applying formal knowledge frame may cue technical vocabulary and principles communicated by authorities such as the instructor or textbook. Several case studies demonstrate that the epistemological framing activated by a particular instructional activity, such as a group discussion or an exam, may be an important factor in determining when certain conceptual resources are, or are not, activated $[7,41]$. Following this line of reasoning, it may be the case that predict-style and explain-style questions communicate different things about what counts as an answer, which in turn cues different kinds of conceptual resources.

Our current study is specific to predict-style and explainstyle questions about wave mechanics. Whether similar patterns emerge in different content areas (e.g., electrostatics or thermodynamics) is a question we are exploring in emerging work. We hypothesize that the categories we have articulated in the context of wave mechanics are specific instances of more general categories corresponding to the predict-style and explain-style structure of questions across physics content areas. Extending this work to new physics content areas may clarify whether there are nuances to the question structure or features of the physics content itself that contribute to the patterns of responses we see in this study.

\footnotetext{
${ }^{5} \mathrm{An}$ epistemological frame is the set of epistemological resources activated for a particular situation, and answers the question "What is it that's going on here?" [2].
} 
Our results suggest questions about the extent to which these patterns would be impacted by small changes to the instruction that students receive before answering our questions or to the wording of our questions. For example, at University D, a tutorial [42] that students completed inclass prior to answering our superposition questions emphasizes a predictive model and procedures for determining the shape of a spring or string during reflection. In a course taught by author LMG (not included in this data) that more explicitly discussed mechanisms for reflection (in addition to procedural tutorials), we saw that a higher fraction of students used FME resources to answer a question like the cups question- though students still used RP resources more frequently. However, these results may also be explained by small changes in the wording of the question: for example, these students were asked to predict (and draw) the shape of a spring at several instants during a fixed end, and then to "explain why their drawings make sense." It is possible that this wording cued framings like those cued by explain-style questions for some students, and thus a higher fraction of students used FME resources (either instead of, or in addition to, RP resources) to answer the question.

As we have discussed in Sec. III, our study samples from universities that disproportionately serve white and wealthy students as compared to the U.S. college-age population. Though this imbalance is consistent with a broader trend in physics education research, it represents an important limitation to the generalizability of our results. The broader trend leads to a skewed accrual of the benefits of PER to white, wealthy students. Our knowledge of the demographics and representativeness of our sample is limited by the fact that we do not know the demographic information of the courses in which we administered our questions, so we rely on the not-yet-substantiated assumption that the demographics of these physics courses match the overall demographics of the university. In future work we intend to collect data from a more representative sample. We also intend to collect demographic data for the courses we sample from, rather than use university-level data to estimate the demographics of our sample.

\section{INSTRUCTIONAL IMPLICATIONS}

Both RP and FME resources represent types of physics reasoning that have been emphasized in physics education literature. Identifying and mapping appropriate physics and math concepts to a particular problem or translating qualitative problem statements into more quantitative or mathematical forms, as students are doing in RP answers, is an important step in physics problem solving (e.g., Ref. [43]). Identifying relevant entities and activities for the mechanisms of wave mechanics, and chaining them together to construct causal or mechanistic explanations, as students do in FME responses, are important features of mechanistic reasoning [10]. The RP and FME categories, then, call instructors' attention to categories of reasoning that represent "wonderful ideas" [44].

The bar graphs in Figs. 12-17 contribute useful pedagogical content knowledge [45] that instructors can use to guide their choice of in-class or assessment questions that match their goals. Predict-style questions offer the advantage of commonly cuing resources that are appropriate for making specific predictions and are closely tied to the content of the question. Such questions may be useful for assessing students' knowledge of specific mathematical or conceptual models. explain-style questions commonly elicit a broad variety of resources and responses, which would be appropriate for goals of debating ideas, broadening and connecting resources, and sense making and mechanistic reasoning. Though these results are limited to the content area of wave propagation, we hypothesize that predict-style and explain-style questions in other content areas may also elicit different kinds of thinking in the classroom.

It is common in physics teaching and PER to use predictstyle questions to assess student understanding. For example, conceptual inventories such as the Force Concept Inventory [46] or Waves Diagnostic Test [21] almost exclusively use predict-style questions. Our results demonstrate that such assessments may be limited in their capacity to assess students' ability to use the fundamental physics ideas of force, energy, and motion to sense make about complex physics questions. Our results suggest that incorporating explain-style questions into assessments could expand the scope of physics thinking that is valued through classroom discussions or assessment.

\section{ACKNOWLEDGMENTS}

We wish to thank Stephen Kanim, Andrew Elby, Sarah B. McKagan, and Fred Goldberg for their helpful feedback throughout this project. We also wish to thank instructors who helped us to collect the data for this study in their courses. This material is based upon work supported by National Science Foundation Grants No. 1608510, No. 160822, No. 1914603, and No. 1914572, and by the National Science Foundation Graduate Research Fellowship Program under Grant No. 1256082. Any opinions, findings, and conclusions or recommendations expressed in this material are those of the authors and do not necessarily reflect the views of the National Science Foundation. 
[1] D. Hammer, Student resources for learning introductory physics, Am. J. Phys. 68, S52 (2000).

[2] E. F. Redish, A theoretical framework for physics education research: Modeling student thinking, in The Proceedings of the Enrico Fermi Summer School in Physics, edited by E. F. Redish and M. Vincentini (Varenna, Italy, 2003), pp. 1-50, https://eric.ed.gov/?id=ED493138.

[3] R. E. Scherr, Modeling student thinking: An example from special relativity, Am. J. Phys. 75, 272 (2007).

[4] J. P. Smith, A. A. DiSessa, and J. Roschelle, Misconceptions reconceived: A constructivist analysis of knowledge in transition, J. Learn. Sci. 3, 205 (1993).

[5] A. A. diSessa, Toward an epistemology of physics, Cognit. Instr. 10, 105 (1993).

[6] D. Hammer, A. Elby, R. E. Scherr, and E. F. Redish, Resources, framing, and transfer, in Transfer of Learning from a Modern Multidisciplinary Perspective, edited by J.P. Mestre (Information Age Publishing, Greenwich, CT, 2005), p. 89.

[7] L. Lising and A. Elby, The impact of epistemology on learning: A case study from introductory physics, Am. J. Phys. 73, 372 (2005).

[8] J.F. Wagner, The Construction of Similarity: Context Sensitivity and the Transfer of Mathematical Knowledge (University of California, Berkeley, CA, 2003).

[9] L. M. Goodhew, A. D. Robertson, P. R. L. Heron, and R. E. Scherr, Student conceptual resources for understanding mechanical wave propagation, Phys. Rev. Phys. Educ. Res. 15, 020127 (2019).

[10] R. S. Russ, R. E. Scherr, D. Hammer, and J. Mikeska, Reasoning in student scientific inquiry: A framework for discourse analysis developed from philosophy of science, Sci. Educ. 92, 499 (2008).

[11] D. Brown and D. Hammer, Conceptual change in physics, in International Handbook of Research on Conceptual Change, edited by S. Vosniadou (Routledge, London, 2008), pp. 140-149.

[12] L. M. Goodhew, A. D. Robertson, P. R. L. Heron, and R. E. Scherr, Student conceptual resources for understanding mechanical wave propagation, Phys. Rev. Phys. Educ. Res. 15, 020127 (2019).

[13] H. C. Sabo, L. M. Goodhew, and A. D. Robertson, University student conceptual resources for understanding energy, Phys. Rev. Phys. Educ. Res. 12, 010126 (2016).

[14] B. W. Harrer, V. J. Flood, and M. C. Wittmann, Productive resources in students' ideas about energy: An alternative analysis of Watts' original interview transcripts, Phys. Rev. ST Phys. Educ. Res. 9, 023101 (2013).

[15] A. J. Richards, D. C. Jones, and E. Etkina, How students combine resources to make conceptual breakthroughs, Res. Sci. Educ. 50, 1119 (2020).

[16] D. Hammer and A. Elby, Tapping epistemological resources for learning physics, J. Learn. Sci. 12, 53 (2003).

[17] B. W. Harrer, Identifying productive resources in secondary school students' discourse about energy, Doctoral thesis, University of Maine, 2013.

[18] L. C. McDermott, A view from physics, in Toward a Scientific Practice of Science Education, edited by M. Gardner, J. G. Greeno, F. Reif, A. Schoenfeld, A. A.
diSessa, and E. Stage (Lawrence Erlbaum Associates, Hillsdale, NJ, 1990), pp. 3-30.

[19] E. C. Sayre and M. C. Wittmann, Plasticity of intermediate mechanics students' coordinate system choice, Phys. Rev. ST Phys. Educ. Res. 4, 020105 (2008).

[20] M. Kryjevskaia, M. R. Stetzer, and P. R. L. Heron, Student understanding of wave behavior at a boundary: The limiting case of reflection at fixed and free ends, Am. J. Phys. 79, 508 (2011).

[21] M. C. Wittmann, Making sense of how students come to an understanding of physics: An example from mechanical waves, Doctoral thesis, University of Maryland, 1998.

[22] S. Kanim and X.C. Cid, Demographics of physics education research, Phys. Rev. Phys. Educ. Res. 16, 020106 (2020).

[23] G. Gay, Culturally Responsive Teaching: Theory, Research, and Practice, 2nd ed. (Teachers College Press, New York, NY, 2012).

[24] D. B. Martin, Researching race in mathematics education, Teachers College Record 111, 295 (2009), https://www .tcrecord.org/Content.asp?ContentId=15226.

[25] P. Mulvey (private communication).

[26] R. Mondesir and A. D. Robertson, Towards characterizing the demographics of introductory physics courses, in Proceedings of the 2020 Physics Education Research Conference, virtual conference, edited by S. Wolf, E. Bennett, and B. Frank (AIP, New York, 2020).

[27] K. Rosa and F. M. Mensah, Educational pathways of black women physicists: Stories of experiencing and overcoming obstacles in life, Phys. Rev. Phys. Educ. Res. 12, 020113 (2016).

[28] S. Hyater-Adams, C. Fracchiolla, T. Williams, N. Finkelstein, and K. Hinko, Deconstructing black physics identity: Linking individual and social constructs using the critical physics identity framework, Phys. Rev. Phys. Educ. Res. 15, 020115 (2019).

[29] Z. Hazari, Doing physics and being other, in Proceedings of the American Association of Physics Teachers Summer Meeting (2020).

[30] I. Rodriguez and B. Zamarripa Roman, Dos Neplanteras in Physics/Education/Research, in American Association of Physics Teachers Summer Meeting, Provo, UT (2019).

[31] X. R. Quichocho, E. M. Schipull, and E. W. Close, Understanding physics identity development through the identity performances of black, indigenous, and women of color and LGBTQ + women in physics, in Proceedings of the 2020 Physics Education Research Conference, virtual conference, edited by S. Wolf, M. Bennett, and B. Frank (AIP, New York, 2020), pp. 412-417.

[32] G. L. Cochran, A. Gupta, S. A. Hyater-Adams, A. V. Knaub, and B.Z. Roman, Emerging reflections from the people of color (POC) at PERC discussion space, arXiv:1907.01655v2.

[33] Equality of Opportunity Project, https://equality-ofopportunity.org/data.

[34] J. Cohen, A coefficient of agreement for nominal scales, Educ. Psychol. Meas. 20, 37 (1960).

[35] J. R. Landis and G. G. Koch, The measurement of observer agreement for categorical data, Biometrics 33, 159 (1977). 
[36] M. L. McHugh, Lessons in biostatistics interrater reliability: The kappa statistic, Biochem. Medica 22, 276 (2012).

[37] R. S. Russ, J. E. Coffey, D. Hammer, and P. Hutchison, More accountable to scientific reasoning: A case for attending to mechanistic thinking, Sci. Educ. 93, 875 (2009).

[38] T.D. Cook, Randomized experiments in education: Why are they so rare?, Educ. Eval. Policy Anal. 24, 175 (2002).

[39] L. C. McDermott, Millikan lecture 1990: What we teach and what is learned-closing the gap, Am. J. Phys. 59, 301 (1991).

[40] G. Posner, K. Strike, P. Hewson, and W. Gertzog, Accommodation of a scientific conception: Toward a theory of conceptual change, Sci. Educ. 66, 211 (1982).
[41] J. Tuminaro and E. F. Redish, Elements of a cognitive model of physics problem solving: Epistemic games, Phys. Rev. ST Phys. Educ. Res. 3, 020101 (2007).

[42] L. C. McDermott and P. S. Shaffer, Tutorials in Introductory Physics (Pearson Learning Solutions, 2002).

[43] B. R. Wilcox, M.D. Caballero, D. A. Rehn, and S. J. Pollock, Analytic Framework for Students' Use of Mathematics in Upper-Division Physics, Phys. Rev. ST Phys. Educ. Res. 9, 020119 (2013).

[44] E. Duckworth, The Having of Wonderful Ideas and Other Essays on Teaching and Learning (Teachers College Press, New York, 1987), pp. 1-14.

[45] L. S. Shulman, Those who understand: Knowledge growth in teaching, Educ. Res. 15, 4 (1986).

[46] D. Hestenes, M. Wells, and G. Swackhamer, Force Concept Inventory, Phys. Teach. 30, 141 (1992). 